\title{
OPERATIONS STOCHASTIQUES DE CAPITALISATION
}

\author{
By Pierre Devolder
}

Royale Belge, Bruxelles

\begin{abstract}
This paper presents a stochastic model of capitalization which takes into account the financial risk in the actuarial processes.

We first introduce a stochastic differential equation which allows us to define the capitalization and actualization processes.

We use these concepts to present a new principle of premium calculation for the capitalization operations, based on the equality between backward reserve and conditional expectation of the forward reserve.

A generalization of the classical Thiele equation in life insurance is also given.

Numerical examples illustrate the model.
\end{abstract}

\section{INTRODUCTION}

La théorie classique des opérations financières et viagères est basée sur une hypothèse de constance des taux d'intérêt.

Or la conjoncture économique montre que les conditions de capitalisation peuvent fluctuer considérablement dans le temps; d'autre part, si les taux d'intérêt varient dans le temps, ils présentent également un facteur d'incertitude quant à leur évolution future.

Il est donc naturel de tenter de modéliser le phénomène de capitalisation par un processus stochastique.

Divers modèles stochastiques en temps discret ont déjà été développés en se basant par exemple sur un modèle auto-régressif (PollaRD, 1971), ou sur une chaîne de Markov (SCHNIEPER, 1983).

Il semblait intéressant de généraliser cette approche en temps continu. Pour ce, un outil naturel, déjà utilisé de manière systématique en économie et en finances est l'intégration stochastique de ITO (cf. MALliaris and Brock, 1981).

Nous nous proposons ici d'appliquer ce type de modélisation au calcul financier et viager.

Nous introduisons un processus de taux (Flux d'intérêt wienérien), construit à partir d'une intégrale stochastique et étudions l'impact de l'incertitude financière ainsi introduite sur les primes et les réserves mathématiques des opérations de capitalisation et d'assurance sur la vie. Nous définissons en particulier une tarification stochastique où les primes annuelles jouent le rôle de variables de contrôle impulsionnel sur l'état du système représenté par la réserve mathématique.

Nous généralisons également l'équation différentielle de Thiele de la réserve mathématique des opérations viagères, en une équation différentielle stochastique. 
L'approche utilisée permet d'étalonner le risque financier, de retrouver comme cas limite le modèle déterministe classique et d'obtenir une tarification facilement calculable où l'influence du risque financier peut être isolée.

Des exemples numériques illustrent le modèle.

\section{FLUX D'INTERET WIENERIEN}

Considérons un capital $C(0)>0$, capitalisé à un taux d'intérêt instantané constant $\delta$.

On sait que la valeur capitalisée de $C(0)$ à l'instant $t$ est donnée par: $C(t)=$ $C(0) \exp \delta t$,

$$
\begin{array}{ll}
\text { relation équivalente à: } & C^{\prime}(t)=\delta C(t) \\
\text { ou sous forme intégrale: } & C(t)=C(0)+\int_{0}^{t} C(s) \delta d s .
\end{array}
$$

Le but de ce paragraphe est de généraliser cette équation intégrale, de manière à tenir compte à la fois de la variation dans le temps des taux, et de l'incertitude de leur évolution.

L'équation stochastique de capitalisation étudiée aura la forme:

$$
C(t)=C(0)+\int_{0}^{t} C(s) d I(s)
$$

où $I$ est un processus stochastique, appelé flux d'intérêt.

On se limitera ici aux définitions et propriétés fondamentales utilisées dans les applications financières, renvoyant aux références, pour les démonstrations des résultats classiques de l'intégration stochastique.

\subsection{Equation Différentielle Stochastique de Capitalisation}

Soit $(\Omega, \mathscr{A}, \mathbb{P})$ un espace de probabilité, muni d'une filtration $\left\{\mathscr{F}_{t}, t \geqslant 0\right\}$, c'est-àdire d'une famille croissante de sous $\sigma$-algèbres de $\mathscr{A}$.

Rappelons qu'un processus stochastique $\left\{w(t, \omega) t \in \mathbb{R}^{+}, \omega \in \Omega\right\}$ est un processus de Wiener si:

(a) w est un processus à accroissements indépendants et stationnaires

(b) $w(t)$ admet une distribution normale, $\forall t>0$

(c) $w(0)=0$

(d) $\forall t>0: \mathbb{E}(w(t))=0$

$$
\operatorname{Var}(w(t))=t
$$

Le processus wienérien $w$ est dit adapté à la filtration $\left\{\mathscr{F}_{t}\right\}$ si $w(t)$ est une variable aléatoire $\mathscr{F}_{t}$-mesurable, et ce pour tout $t \geqslant 0$.

Considérons d'autre part une fonction $\delta$ de $[0, T]$ dans $\mathbb{R}^{+}$, et une fonction $\sigma$ de $[0, T]$ dans $\mathbb{R}$, supposées continues par morceaux sur $[0, T]$, telles que $\int_{0}^{T} \delta(s) d s<+\infty$ et $\int_{0}^{T} \sigma^{2}(s) d s<+\infty$. 
A l'aide du processus wienérien, et des fonctions $\delta$ et $\sigma$, on construit un processus stochastique financier, appelé flux d'intérêt wienérien, donné par:

$$
I(t)=\int_{0}^{t} \delta(s) d s+\int_{0}^{t} \sigma(s) d w(s) .
$$

Le premier terme, $I_{d}(t)=\int_{0}^{t} \delta(s) d s$ est un flux déterministe censé ajuster l'évolution moyenne des taux d'intérêt ("trends").

Le second terme, $I_{a}(t)=\int_{0}^{t} \sigma(s) d w(s)$ est une intégrale stochastique par rapport à un processus wienérien (calcul stochastique de ITO, voir, GINMAN and SKOROHOD, 1972), et joue le rôle de perturbation aléatoire ("bruit"), permettant de prendre en compte les variations des taux d'intérêt autour de leur valeur moyenne.

La fonction $\delta$ sera appelée "taux instantané moyen".

La fonction $\sigma$ sera appelée "coefficient de diffusion financière".

Le flux wienérien $I$ satisfait aux propriétés suivantes:

(a) $\mathbb{E}(I(t))=\int_{0}^{t} \delta(s) d s$.

(b) $\operatorname{var}(I(t))=\int_{0}^{t} \sigma^{2}(s) d s$.

(c) Le processus $\{I(t), t>0\}$ est adapté à la filtration $\left\{\mathscr{F}_{\ell}, t>0\right\}$ et admet une différentielle stochastique:

$$
d I(s)=\delta(s) d s+\sigma(s) d w(s) .
$$

On considère alors un capital initial $C(0)>0$ placé sous le flux wienérien $I$, et évoluant suivant l'équation différentielle stochastique:

$$
C(t)=C(0)+\int_{0}^{t} C(s) d I(s)
$$

ou

$$
C(t)=C(0)+\int_{0}^{t} C(s) \delta(s) d s+\int_{0}^{t} C(s) \sigma(s) d w(s)
$$

ou encore sous forme différentielle:

$$
d C(t)=C(t) \delta(t) d t+C(t) \sigma(t) d w(t) .
$$

A propos des équations différentielles stochastiques, on a le théorème d'existence et d'unicité suivant:

THeOREMe 1: On considère l'équation différentielle stochastique:

$$
\left\{\begin{array}{l}
d \xi(t)=a(t, \xi(t)) d t+\sigma(t, \xi(t)) d w(t) \\
\xi(0)=\xi_{0}
\end{array}\right.
$$

où

(a) w est un processus de Wiener, adapté à une filtration $\left\{\mathscr{F}_{s}, s>0\right\}$ 
(b) $\xi_{0}$ est une variable aléatoire $\mathscr{F}_{0}$-mesurable

(c) a $(\cdot, \cdot)$ et $\sigma(\cdot, \cdot)$ sont 2 fonctions de $[0, T] \times \mathbb{R}$ dans $\mathbb{R}$, mesurables en $(t, x)$ satisfaisant:

$$
\begin{gathered}
|a(t, x)|^{2}+|\sigma(t, x)|^{2} \leqslant K\left(1+|x|^{2}\right) \\
|a(t, x)-a(t, y)|^{2}+|\sigma(t, x)-\sigma(t, y)|^{2} \leqslant C\left(|x-y|^{2}\right) .
\end{gathered}
$$

Alors l'équation (1.3) admet une solution $\{\xi(t), t \in[0, T]\}$ adaptée à la filtration $\left\{\mathscr{F}_{t}\right\}$, unique au sens suivant: si $\xi_{1}$ et $\xi_{2}$ sont solutions:

$$
\mathbb{P}\left[\sup _{0 \leq t \leq T}\left|\xi_{1}(t)-\xi_{2}(t)\right|>0\right]=0 .
$$

Démonstration. Voir, Ginman and Skorohod, 1972.

On a donc:

Proposition 1. Sous un flux wienérien, l'équation stochastique de capitalisation (1.2) admet une solution "unique", donnée par la formule explicite:

$$
C(t)=C(0) \cdot \exp \left(\int_{0}^{t}\left(\delta(s)-\frac{1}{2} \sigma^{2}(s)\right) d s+\int_{0}^{t} \sigma(s) d w(s)\right) \text { p.s. }
$$

DÉmonstration. L'existence et l'unicité résultent directement du théorème 1, en prenant:

$$
\begin{aligned}
& a(t, x)=\delta(t) x \\
& \sigma(t, x)=\sigma(t) x
\end{aligned}
$$

Pour obtenir la formule explicite (1.4), on utilise la formule de ITO (voir, Malliaris and Brock, 1981): si un processus $(\xi(t), t \geqslant 0)$ admet une différentielle stochastique

$$
d \xi(t)=a(t) d t+b(t) d w(t)
$$

et si $f(\cdot, \cdot)$ est une fonction de $[0, T] \times \mathbb{R}$ dans $\mathbb{R}$, continue, ainsi que ses dérivées partielles, $\partial f / \partial t, \partial f / \partial x, \partial^{2} f / \partial x^{2}$, alors le processus composé $(f(t, \xi(t)), t \geqslant 0)$ admet une différentielle stochastique donnée par:

$$
\begin{aligned}
d f(t, \xi(t))= & {\left[\frac{\partial f}{\partial t}(t, \xi(t))+\frac{\partial f}{\partial x}(t, \xi(t)) a(t)+\frac{1}{2} \frac{\partial^{2} f}{\partial x^{2}}(t, \xi(t)) b^{2}(t)\right] d t } \\
& +\frac{\partial f}{\partial x}(t, \xi(t)) b(t) \cdot d w(t) .
\end{aligned}
$$

En appliquant cette formule au processus $\left(I^{*}(t), t \in[0, T]\right)$ défini par $I^{*}(t)=$ $\ln C(t)$, on a:

$$
d I^{*}(t)=\left(\delta(t)-\frac{1}{2} \sigma^{2}(t)\right) d t+\sigma(t) d w(t)
$$


ou

$$
I^{*}(t)=I^{*}(0)+\int_{0}^{t}\left(\delta(s)-\frac{1}{2} \sigma^{2}(s)\right) d s+\int_{0}^{t} \sigma(s) d w(s) .
$$

En revenant au capital,

$$
C(t)=C(0) \exp \left(\int_{0}^{t}\left(\delta(s)-\frac{1}{2} \sigma^{2}(s)\right) d s+\int_{0}^{t} \sigma(s) d w(s)\right)
$$

\section{Remarques 1.}

(1) Lorsque $\sigma \equiv 0$, on retrouve le modèle déterministe classique:

$$
C_{d}(t)=C(0) \cdot \exp \int_{0}^{t} \delta(s) d s
$$

où $\delta(\cdot)$ est le taux instantané d'intérêt.

En particulier, si $\delta(\cdot)$ est constant, on obtient la formule classique de capitalisation à intérêts composés:

$$
C_{d}(t)=C(0) \cdot e^{\delta t}=C(0) \cdot u^{t}=C(0) \cdot(1+i)^{t}
$$

(voir, SAADA, 1979).

(2) La perturbation stochastique introduite par ce modèle est donc:

$$
\alpha(t)=\exp \left(\int_{0}^{t} \sigma(s) d w(s)-\frac{1}{2} \int_{0}^{t} \sigma^{2}(s) d s\right)
$$

puisque:

$$
C(t)=C_{d}(t) \cdot \alpha(t)
$$

(3) Soit $s \leqslant t \leqslant T$. On a:

$$
\begin{aligned}
& C(t)= C(0) \exp \int_{0}^{t}\left(\delta(r)-\frac{1}{2} \sigma^{2}(r)\right) d r \cdot \exp \int_{0}^{t} \sigma(r) d w(r) \\
&= C(0) \exp \int_{0}^{s}\left(\delta(r)-\frac{1}{2} \sigma^{2}(r)\right) d r \cdot \exp \int_{0}^{s} \sigma(r) d w(r) \\
& \cdot \exp \int_{s}^{t}\left(\delta(r)-\frac{1}{2} \sigma^{2}(r)\right) d r \cdot \exp \int_{s}^{t} \sigma(r) d w(r) \\
& C(t)=C(s) \cdot \exp \int_{s}^{t}\left(\delta(r)-\frac{1}{2} \sigma^{2}(r)\right) d r \cdot \exp \int_{s}^{t} \sigma(r) d w(r) \\
& C(t)=C(s) \cdot C_{s}(t)
\end{aligned}
$$

$C_{s}(t)=\exp \int_{s}^{t}\left(\delta(r)-\frac{1}{2} \sigma^{2}(r)\right) d r \cdot \exp \int_{s}^{t} \sigma(r) d w(r)$ est appelé facteur de capitalisation de $s$ vers $t(s \leqslant t)$. Financièrement, $C_{s}(t)$ peut s'interpréter comme valeur acquise en $t$, d'un capital unitaire placé en $s$. 
(4) On a:

$$
C_{s}(t)=\exp \int_{s}^{t} \delta(r) d r \cdot \alpha_{s}(t)
$$

$\alpha_{s}(t)$ sera appelé perturbation stochastique sur $(s, t)$.

\subsection{Processus d'actualisation}

On peut également introduire le coefficient d'actualisation vers le passé.

Soit toujours $s<t$. On a:

$$
C(t)=C(s) \cdot \exp \left(\int_{s}^{t}\left(\delta(r)-\frac{1}{2} \sigma^{2}(r)\right) d r\right) \cdot \exp \int_{s}^{t} \sigma(r) d w(r)
$$

ou:

$$
C(s)=C(t) \cdot \exp \left(\int_{s}^{t}\left(\frac{1}{2} \sigma^{2}(r)-\delta(r)\right) d r\right) \cdot \exp \int_{s}^{t}-\sigma(r) d w(r) .
$$

Le facteur d'actualisation stochastique de $t$ vers $s$, noté $\varphi_{s}(t)$, est donc:

$$
\varphi_{s}(t)=\frac{1}{C_{s}(t)}=\exp \left(\int_{s}^{t}\left(\frac{1}{2} \sigma^{2}(r)-\delta(r)\right) d r\right) \cdot \exp \int_{s}^{t}-\sigma(r) d w(r) .
$$

Dans le cas déterministe $(\sigma \equiv 0)$, on a:

$$
\varphi_{s}(t)=\exp -\int_{s}^{t} \delta(r) d r .
$$

\subsection{Equation Différentielle de Kolmogorov et Moments}

A l'équation différentielle stochastique de capitalisation:

$$
d C(t)=\delta(t) C(t) d t+\sigma(t) C(t) d w(t)
$$

est naturellement associé l'opérateur différentiel

$$
\mathscr{D}=\delta(t) x \frac{\partial}{\partial x}+\frac{1}{2} \sigma^{2}(t) x^{2} \frac{\partial^{2}}{\partial x^{2}}
$$

défini sur l'espace des fonctions de 2 variables réelles, $u(t, x)$, deux fois continûment différentiables par rapport à la variable spatiale $x$.

Plus précisément, soit $f(x)$ une fonction deux fois continûment différentiable, telle qu'il existe $K>0, m>0$ :

$$
|f(x)|+\left|f^{\prime}(x)\right|+\left|f^{\prime \prime}(x)\right| \leqslant K\left(1+|x|^{m}\right) .
$$

Soit, d'autre part: $p(s, x, t, \Gamma)=P(C(t) \in \Gamma \mid C(s)=x)$ les probabilités de transition du processus de capitalisation défini par l'équation (1.2). 
En notant:

$$
u(s, x)=\mathbb{E}_{s x}(f(C(t)))=\int f(y) p(s, x, t, d y) \quad(s<t, t \text { fixé }),
$$

on a le théorème suivant (voir: GINMAN and SKOROHOD, 1972):

Theoreme 2. Si les fonctions $\delta$ et $\sigma$ sont continues, la fonction $u(\cdot, \cdot)$ possède des dérivées première et seconde par rapport à $x$, continues en $s$ et $x$, est dérivable par rapport à s et vérifie l'équation aux dérivées partielles parabolique:

$$
\frac{\partial u}{\partial s}(s, x)+\delta(s) x \frac{\partial u}{\partial x}(s, x)+\frac{1}{2} \sigma^{2}(s) x^{2} \frac{\partial^{2} u}{\partial x^{2}}(s, x)=0
$$

avec comme condition aux limites: $\lim _{s \uparrow t} u(s, x)=f(x)$.

Ce théorème permet d'obtenir facilement les moments des processus de capitalisation et d'actualisation.

Proposition 2. Soient $C_{s}(t)$ et $\varphi_{s}(t)$, les facteurs de capitalisation et d'actualisation de $s$ vers $t$ définis en (1.7) et (1.9). On a:

(i) $\mathbb{E}\left(C_{s}(t) \mid \mathscr{F}_{s}\right)=\exp \int_{s}^{t} \delta(r) d r$

(ii) $\operatorname{var}\left(C_{s}(t) \mid \mathscr{F}_{s}\right)=\exp \int_{s}^{t} 2 \delta(r) d r\left(\exp \int_{s}^{t} \sigma^{2}(r) d r-1\right)$

(iii) $\mathbb{E}\left(\varphi_{s}(t) \mid \mathscr{F}_{s}\right)=\exp \int_{s}^{t}\left(\sigma^{2}(r)-\delta(r)\right) d r$

$$
\text { (iv) } \operatorname{var}\left(\varphi_{s}(t) \mid \mathscr{F}_{s}\right)=\exp \int_{s}^{t}\left(2 \sigma^{2}(r)-2 \delta(r)\right) d r\left(\exp \int_{s}^{t} \sigma^{2}(r) d r-1\right)
$$

Démonstration. Supposons d'abord les fonctions $\delta$ et $\sigma$ continues; on peut alors utiliser le théorème 2 :

(i) en prenant $f(x)=x$, on a:

$$
\begin{aligned}
u(s, x) & =\mathbb{E}_{s x}(C(t))=x \mathbb{E}\left[C_{s}(t) \mid \mathscr{F}_{s}\right] \\
& =x \exp \int_{s}^{t}\left(\delta(r)-\frac{1}{2} \sigma^{2}(r)\right) d r \cdot \mathbb{E}\left(\exp \int_{s}^{t} \sigma(r) d w(r)\right) .
\end{aligned}
$$

Notons: $\psi(s)=\mathbb{E}\left(\exp \int_{s}^{t} \sigma(r) d w(r)\right)$.

La fonction $u(\cdot, \cdot)$ étant solution de l'équation (1.11), on a:

$$
\begin{aligned}
\frac{\partial u}{\partial x}(s, x)= & \exp \int_{s}^{t}\left(\delta(r)-\frac{1}{2} \sigma^{2}(r)\right) d r \cdot \psi(s) \\
\frac{\partial u}{\partial s}(s, x)= & -x\left(\delta(s)-\frac{1}{2} \sigma^{2}(s)\right) \exp \int_{s}^{t}\left(\delta(r)-\frac{1}{2} \sigma^{2}(r)\right) d r \cdot \psi(s) \\
& +x \exp \int_{s}^{t}\left(\delta(r)-\frac{1}{2} \sigma^{2}(r)\right) d r \cdot \frac{d \psi(s)}{d s}
\end{aligned}
$$


En replaçant dans (1.11), on obtient l'équation différentielle:

$$
\frac{1}{2} \sigma^{2}(s) \psi(s)+\frac{d \psi}{d s}(s)=0
$$

avec comme condition aux limites $\psi(t)=1$, i.e.

$$
\psi(s)=\exp \left(\frac{1}{2} \int_{s}^{t} \sigma^{2}(r) d r\right)
$$

Finalement,

$$
\begin{aligned}
u(s, x) & =x \exp \int_{s}^{t}\left(\delta(r)-\frac{1}{2} \sigma^{2}(r)\right) d r \cdot \exp \left(\frac{1}{2} \int_{s}^{t} \sigma^{2}(r) d r\right) \\
& =x \exp \int_{s}^{t} \delta(r) d r .
\end{aligned}
$$

Donc

$$
\mathbb{E}\left[C_{s}(t) \mid \mathscr{F}_{s}\right]=\exp \int_{s}^{t} \delta(r) d r
$$

(ii) se démontre d'une manière analogue, en prenant $f(x)=x^{2}$.

(iii) On a:

$$
\begin{aligned}
\mathbb{E}\left[\varphi_{s}(t) \mid \mathscr{F}_{s}\right] & =\exp \int_{s}^{t}\left(\frac{1}{2} \sigma^{2}(r)-\delta(r)\right) d r \cdot \mathbb{E}\left[-\int_{s}^{t} \sigma(r) d w(r)\right] \\
& =\exp \int_{s}^{t}\left(\frac{1}{2} \sigma^{2}(r)-\delta(r)\right) d r \cdot \mathbb{E}\left[\int_{s}^{t}(-\sigma(r)) d w(r)\right] \\
& =\exp \int_{s}^{t}\left(\frac{1}{2} \sigma^{2}(r)-\delta(r)\right) d r \cdot \exp \int_{s}^{t} \frac{1}{2}(-\sigma(r))^{2} d r \operatorname{par}(1.16) \\
& =\exp \int_{s}^{t}\left(\sigma^{2}(r)-\delta(r)\right) d r .
\end{aligned}
$$

(iv) se démontre d'une manière analogue en partant de la variance du processus de capitalisation.

Dans le cas général où $\delta$ et $\sigma$ sont simplement continues par morceaux, il suffit d'appliquer l'équation (1.11) sur les intervalles successifs de continuité commune de $\delta$ et $\sigma$.

\section{ReMARQues 2.}

(1) La formule (i) montre que l'espérance mathématique du facteur de capitalisation stochastique $C_{s}(t)$ est égale au facteur de capitalisation déterministe classique, $\exp \int_{s}^{t} \delta(r) d r$.

L'introduction de la diffusion financière joue donc bien le rôle de perturbation autour d'une valeur moyenne déterministe. 
(2) La formule (iii) par contre montre que l'espérance mathématique du facteur d'actualisation stochastique $\varphi_{s}(t)$ est supérieur au facteur d'actualisation déterministe classique, $\exp \int_{s}^{t}-\delta(r) d r$.

$$
\mathbb{E}\left[\varphi_{s}(t) \mid \mathscr{F}_{s}\right]=\exp \int_{s}^{t}-\delta(r) d r \cdot \exp \int_{s}^{t} \sigma^{2}(r) d r .
$$

En fait, tout se passe comme si le taux instantané moyen $\delta$ était remplacé par un taux diminué du risque financier, donné par $\delta-\sigma^{2}$.

La fonction $\delta$ sera appelée taux instantané avec risque.

La fonction $\delta-\sigma^{2}$ sera appelée taux instantané hors risque.

\section{OPERATIONS DE CAPITALISATION}

Ce paragraphe a pour objet de présenter une approche stochastique de la tarification et de la politique de réservation des opérations de capitalisation, et d'analyser l'influence du risque financier sur ces éléments.

\subsection{Définition et Approche Déterministe Classique}

Un contrat de capitalisation est un contrat qui prévoit, contre versement d'une prime unique ou de plusieurs primes périodiques, le paiement au terme du contrat, d'un capital fixé.

On traitera ici 2 cas: paiement par prime unique; paiement par primes annuelles.

La durée du contrat sera notée $N$.

Le capital souscrit sera supposé égal à 1 .

On ne s'intéressera ici qu'à des primes pures.

Dans un cadre déterministe, supposons que les taux soient ajustés par le flux: $I_{d}(t)=\int_{0}^{t} \delta(s) d s$.

Si le contrat est payé par prime unique, la prime unique $P^{*}$ est déterminée par l'équation d'équivalence en $t=0$ :

$$
P^{*} \cdot \exp \int_{0}^{N} \delta(s) d s=1
$$

donc

$$
P^{*}=\exp -\int_{0}^{N} \delta(s) d s
$$

La réserve mathématique en $t$ est égale à la valeur capitale en $t$, de la prime unique:

$$
V^{*}(t)=P^{*} \cdot \exp \int_{0}^{t} \delta(s) d s=\exp -\int_{t}^{N} \delta(s) d s .
$$

Si le contrat est alimentée par des primes annuelles nivelées, la prime annuelle 
$p^{*}$ est déterminée par l'équation d'équivalence en $t=0$ :

$$
p^{*} \cdot \sum_{j=0}^{N-1} \exp -\int_{0}^{j} \delta(s) d s=\exp -\int_{0}^{N} \delta(s) d s
$$

donc

$$
p^{*}=\frac{\exp -\int_{0}^{N} \delta(s) d s}{\sum_{j=0}^{N-1} \exp -\int_{0}^{j} \delta(s) d s} .
$$

La réserve mathématique en $t$ est égale à la somme des primes annuelles versées avant l'instant $t$, et capitalisées en $t$ (formule rétrospective):

$$
V^{*}(t)=\sum_{j=0}^{[t]} p^{*} \exp \int_{j}^{t} \delta(s) d s=\frac{\sum_{j=0}^{[t]} \exp \int_{j}^{t} \delta(s) d s}{\sum_{j=0}^{N-1} \exp \int_{j}^{N} \delta(s) d s} .
$$

Cette réserve peut également être définie de manière prospective, comme différence entre la valeur actuelle du capital et valeur actuelle des primes futures:

$$
V^{*}(t)=\exp -\int_{t}^{N} \delta(s) d s-p^{*} \cdot \sum_{j=[t]+1}^{N-1} \exp -\int_{t}^{j} \delta(s) d s .
$$

L'égalité de ces 2 réserves résulte de la définition de $p^{*}$.

\subsection{Evolution Stochastique des Réserves}

Une premiére prise en compte du risque financier de placement consiste à faire évaluer stochastiquement les réserves mathématiques du contrat, sous un flux wienérien $I(t)=\int_{0}^{t} \delta(s) d s+\int_{0}^{t} \sigma(s) d w(s)$, et de calculer les primes sous le flux déterministe moyen correspondant, $I_{d}(t)=\mathbb{E}(I(t))=\int_{0}^{t} \delta(s) d s$.

Examinons successivement les cas d'un paiement par prime unique ou par primes annuelles.

\section{(1) Prime Unique}

La prime unique $P^{*}$ étant donnée par (2.1), la réserve mathématique stochastique à l'instant $t, V(t)$ est la valeur capitalisée de cette prime sous le flux $I$ :

$$
\begin{aligned}
V(t) & =P^{*} \cdot C_{0}(t) \\
& =\exp -\int_{0}^{N} \delta(s) d s \cdot \exp \int_{0}^{t}\left(\delta(s)-\frac{\sigma^{2}(s)}{2}\right) d s \cdot \exp \int_{0}^{t} \sigma(s) d w(s) \\
& =\exp -\int_{t}^{N} \delta(s) d s \cdot \exp \left(\int_{0}^{t} \sigma(s) d w(s)-\int_{0}^{t} \frac{\sigma^{2}(s)}{2} d s\right) \\
& =V^{*}(t) \cdot \alpha(t)
\end{aligned}
$$


On a donc

$$
\mathbb{E}(V(t))=\mathbb{E}\left(V^{*}(t) \cdot \alpha(t)\right)=V^{*}(t)
$$

où $V^{*}(t)$ est la réserve déterministe obtenue en (2.2). Au terme du contrat, la réserve stochastique $V$ sera généralement différente du capital unitaire souscrit, l'écart, de moyenne nulle, étant:

$$
\Delta=1-\exp \left(\int_{0}^{N} \delta(s) d w(s)-\int_{0}^{N} \frac{\sigma^{2}(s)}{2} d s\right) .
$$

\section{(2) Primes Annuelles}

Les primes annuelles $p^{*}$ étant données par (2.3), la réserve mathématique est solution de l'équation différentielle stochastique:

$$
d V(t)=V(t) \delta(t) d t+V(t) \sigma(t) d w(t)+\sum_{j=0}^{N-1} p^{*} \varepsilon_{j}(d t)
$$

où $\varepsilon_{j}(d t)$ est la mesure de Dirac au point $t=j$ (apport de la prime annuelle).

La solution de cette équation s'exprime comme somme des primes déjà versées capitalisées (cf. (2.4)):

$$
\begin{aligned}
V(t) & =\sum_{j=0}^{[t]} p^{*} \cdot C_{j}(t) \\
& =\exp -\int_{0}^{N} \delta(s) d s \cdot \frac{\sum_{j=0}^{[t]} \exp \int_{j}^{t}\left(\delta(s)-\frac{1}{2} \sigma^{2}(s)\right) d s \cdot \exp \int_{j}^{t} \sigma(s) d w(s)}{\sum_{j=0}^{N-1} \exp -\int_{0}^{j} \delta(s) d s} .
\end{aligned}
$$

En comparant à la réserve déterministe $V^{*}(2.4)$ :

$$
\begin{aligned}
\eta(t)=\frac{V(t)}{V^{*}(t)} & =\frac{\sum_{j=0}^{[t]} \exp \left(\int_{j}^{t}\left(\delta(s)-\frac{1}{2} \sigma^{2}(s)\right) d s+\int_{j}^{t} \sigma(s) d w(s)\right)}{\sum_{j=0}^{[t]} \exp \int_{j}^{t} \delta(s) d s} \\
& =\frac{\sum_{j=0}^{[t]} \exp \int_{j}^{t} \delta(s) d s \cdot \alpha_{j}(t)}{\sum_{j=0}^{[t]} \exp \int_{j}^{t} \delta(s) d s}
\end{aligned}
$$

où $\alpha_{j}(t)$ est défini en (1.8).

Ce rapport est donc une moyenne pondérée de perturbations stochastiques.

La moyenne de la réserve stochastique est encore égale à la réserve déterministe:

$$
\mathbb{E}(V(t))=V^{*}(t) \cdot \mathbb{E}\left(\frac{\sum_{j=0}^{[t]} \exp \int_{j}^{t} \delta(s) d s \cdot \alpha_{j}(t)}{\sum_{j=0}^{[t]} \exp \int_{j}^{t} \delta(s) d s}\right)=V^{*}(t) .
$$


Au terme du contrat, la réserve stochastique est donnée par:

$$
V(N)=\frac{\sum_{j=0}^{N-1} \exp -\int_{0}^{j} \delta(s) d s \cdot \alpha_{j}(N)}{\sum_{j=0}^{N-1} \exp -\int_{0}^{j} \delta(s) d s} .
$$

\subsection{Tarification Stochastique}

L'approche stochastique considérée au paragraphe 2.2, bien que tenant compte du risque de placement, n'est pas satisfaisante.

En effet, dans ce cadre:

(1) les réserves mathématiques ne tendent pas presque sûrement vers le capital souscrit;

(2) les primes calculées de manière classique n'incorporent pas le risque financier.

Afin de remédier à ces deux inconvénients, nous nous proposons d'introduire une tarification stochastique et une politique de réservation correspondante.

La situation sera différente, suivant que le financement se fait par prime unique ou primes annuelles.

En effet, en cas de paiement par primes annuelles stochastiques, celles-ci pourront servir de variables de contrôle sur l'état du système modélisé par la réserve mathématique, et être ajustées en cours de contrat, suivant l'évolution des taux.

Par contre, aucun contrôle n'est possible sur la réserve mathématique, en cas de paiement par prime unique.

\subsubsection{Tarification à Prime Unique et Réserve de Risque}

La prime unique stochastique $\tilde{P}$ est définie par l'équation d'équivalence:

$$
\tilde{P} \cdot C_{0}(N)=1
$$

i.e.

$$
\tilde{P} \cdot \exp \int_{0}^{N}\left(\delta(s)-\frac{\sigma^{2}(s)}{2}\right) d s \cdot \exp \int_{0}^{N} \sigma(s) d w(s)=1
$$

Donc

$$
\begin{gathered}
\tilde{P}=\exp -\int_{0}^{N}\left(\delta(s)-\frac{1}{2} \sigma^{2}(s)\right) d s \cdot \exp -\int_{0}^{N} \sigma(s) d w(s) \\
\tilde{P}=P^{*} \cdot \frac{1}{\alpha(N)}
\end{gathered}
$$

où $P^{*}$ est la prime unique déterministe (2.1).

Cette prime $\tilde{P}$ est une variable aléatoire, dépendant de tout le futur du processus. 
Afin de tarifer, en $t=0$, on peut prendre l'espérance mathématique de $\tilde{\boldsymbol{P}}$ :

$$
\begin{aligned}
\mathbb{E} \tilde{P} & =P^{*} \cdot \mathbb{E}\left(\exp \int_{0}^{N} \frac{1}{2} \sigma^{2}(s) d s-\sigma(s) d w(s)\right) \\
& =P^{*} \cdot \exp \int_{0}^{N} \sigma^{2}(s) d s \\
& =P^{*}\left(1+\left(\exp \int_{0}^{N} \sigma^{2}(s) d s-1\right)\right) \\
& =P^{*}(1+\operatorname{var} \alpha(N)) \\
& =\exp \int_{0}^{N}\left(\sigma^{2}(s)-\delta(s)\right) d s
\end{aligned}
$$

La formule (2.13) montre que cette espérance mathématique est supérieure à la prime déterministe, le facteur de pénalisation étant directement lié au risque de placement sur l'horizon $[0, N]$, puisque proportionnel à la variance de la fonction de perturbation stochastique.

La formule (2.14) montre que tout se passe comme si la prime était calculée de manière classique, aux taux hors-risque $\delta-\sigma^{2}$. Quant à la variance de la prime stochastique, on a:

$$
\begin{aligned}
\operatorname{Var} \tilde{P}= & \exp -\int_{0}^{N}\left(2 \delta(s)-\sigma^{2}(s)\right) d s \cdot \operatorname{var}\left(\exp \int_{0}^{N}-\sigma(s) d w(s)\right) \\
= & \exp -\int_{0}^{N}\left(2 \delta(s)-\sigma^{2}(s)\right) d s \\
& \cdot\left(\exp \int_{0}^{N} 2 \sigma^{2}(s) d s-\exp \int_{0}^{N} \sigma^{2}(s) d s\right) \\
= & \exp 2 \int_{0}^{N}\left(\sigma^{2}(s)-\delta(s)\right) d s \cdot\left(\exp \int_{0}^{N} \sigma^{2}(s) d s-1\right) \\
= & (\mathbb{E} \tilde{P})^{2} \cdot \operatorname{var} \alpha(N) .
\end{aligned}
$$

A l'instar de la prime stochastique $\tilde{P}$, introduisons une réserve stochastique en $t, \tilde{V}(t)$ : celle-ci est définie comme étant la prime unique stochastique de l'opération de capitalisation souscrite en $t$, pour une durée $(N-t)$ (approche prospective).

Donc

$$
\tilde{V}(t)=\varphi_{t}(N)=\exp \int_{t}^{N}-\left(\delta(s)-\frac{\sigma^{2}(s)}{2}\right) d s \cdot \exp -\int_{t}^{N} \sigma(s) d w(s) .
$$

La réserve mathématique $V(t)$, sera définie comme l'espérance conditionnelle 
en $t$, de la réserve stochastique $\tilde{V}(t)$ :

$$
\begin{aligned}
V(t) & =\mathbb{E}\left(\tilde{V}(t) \mid \mathscr{F}_{t}\right) \\
& =\exp -\int_{t}^{N}\left(\delta(s)-\frac{\sigma^{2}(s)}{2}\right) d s \cdot \mathbb{E}\left(\exp -\int_{t}^{N} \sigma(s) d w(s)\right) \\
& =\exp -\int_{t}^{N}\left(\delta(s)-\sigma^{2}(s) d s\right. \\
V(t) & =\exp -\int_{t}^{N} \delta(s) d s\left(\exp \int_{t}^{N} \sigma^{2}(s) d s-1+1\right) \\
V(t) & =V^{*}(t) \cdot\left(1+\operatorname{var} \alpha_{t}(N)\right)
\end{aligned}
$$

où $V^{*}(t)$ est la réserve déterministe (2.2).

La réserve obtenue est donc constamment supérieure à la réserve classique; la pénalisation, en pourcentage de la réserve déterministe, est néanmoins une fonction décroissante du temps, nulle au terme du contrat.

Grâce à cette politique, il existe donc une réserve de risque financier, $\varepsilon$, progressivement consommée au cours du contrat, et censée rétribuer l'organisme financier assumant le risque de placement:

$$
V(t)=V^{*}(t)+\varepsilon(t)
$$

avec

$$
\varepsilon(t)=V^{*}(t) \cdot \operatorname{var} \alpha_{t}(N)
$$

En particulier:

$$
\begin{aligned}
\varepsilon(0) & =\exp \left(-\int_{0}^{N}\left(\delta(s)-\sigma^{2}(s)\right) d s-\exp \left(-\int_{0}^{N} \delta(s) d s\right)\right. \\
& \left.=P-P^{*} \quad \text { (surprime payée en } t=0\right) .
\end{aligned}
$$

(b) $\quad \varepsilon(N)=0$.

La réserve $V$ tend donc bien vers la capital souscrit, pour $t \rightarrow N$. D'autre part, les formules (2.14) et (2.17) montrent que l'ensemble de l'opération peut être considéré comme calculé au taux hors-risque $\delta-\sigma^{2}$.

\subsubsection{Tarification à Primes Annuelles et Contrôle}

Nous nous proposons d'établir ici une tarification stochastique à primes annuelles tenant compte du risque financier, où les primes annuelles, qui seront des variables aléatoires, joueront le rôle de contrôle sur la réserve mathématique.

La tarification sera basée sur une condition d'équilibre entre réserves prospective et rétrospective.

Définissons d'abord la notion de tarification mesurable par rapport à une filtration $\left\{\mathscr{F}_{t}\right\}$. 
DÉFINITION. Une tarification mesurable par rapport à une filtration $\left\{\mathscr{F}_{t}\right\}$ est une suite de variables aléatoires $\bar{p}_{0}, \bar{p}_{1}, \ldots, \bar{p}_{N-1}$, payables en $t=0,1, \ldots, N-1$, et telles que:

$$
\forall j \bar{p}_{j} \text { est } \mathscr{F}_{j} \text {-mesurable. }
$$

Chaque prime peut donc être calculée en fonction des informations connues à sa date d'échéance.

La réserve mathématique correspondant à une tarification mesurable $\left(\bar{p}_{0}\right.$, $\left.\bar{p}_{1}, \ldots, \bar{p}_{N-1}\right)$ est définie comme solution de l'équation différentielle stochastique:

$$
d V(t)=V(t) \delta(t) d t+V(t) \sigma(t) d w(t)+\sum_{j=0}^{N-1} \bar{p}_{j} \varepsilon_{j}(d t) .
$$

Les variables aléatoires $\left\{\bar{p}_{j}\right\}$ jouent donc le rôle de contrôles impulsionnels sur la réserve.

La solution de cette équation s'exprime comme somme des primes déjà versées, capitalisées:

$$
V(t)=\sum_{j=0}^{[t]} \bar{p}_{j} C_{j}(t)=\sum_{j=0}^{[t]} \bar{p}_{j} \cdot \exp \int_{j}^{t}\left(\delta(s)-\frac{1}{2} \sigma^{2}(s)\right) d s \cdot \exp \int_{j}^{t} \sigma(s) d w(s) .
$$

Remarquons que le processus $V$ est adapté à la filtration $\left\{\mathscr{F}_{t}\right\}$, par définition de la tarification mesurable. Cette réserve $V$ est une réserve rétrospective.

On peut également introduire une réserve prospective, égale à la différence entre la valeur actualisée du capital et la valeur actualisée des primes futures:

$$
\begin{aligned}
V_{p}(t)= & \varphi_{t}(N)-\sum_{j=[t]+1}^{N-1} \varphi_{t}(j) \bar{p}_{j} \\
= & \left(\exp -\int_{t}^{N}\left(\delta(s)-\frac{1}{2} \sigma^{2}(s)\right) d s \cdot \exp -\int_{t}^{N} \delta(s) d w(s)\right) \\
& -\sum_{j=[t]+1}^{N-1} \bar{p}_{j} \cdot \exp -\int_{t}^{j}\left(\delta(s)-\frac{\sigma^{2}(s)}{2}\right) d s \cdot \exp -\int_{t}^{j} \sigma(s) d w(s) .
\end{aligned}
$$

Contrairement au processus $V$, le processus $V_{p}$ 'est plus adapté à la filtration $\left\{\mathscr{F}_{t}\right\}$.

Pour retrouver la règle déterministe d'égalité des réserves prospectives et rétrospectives, il faudra jouer sur les contrôles $\bar{p}_{j}$, et obtenir une tarification appelée ici tarification d'équilibre.

Pour ce, et dans le but de niveler au mieux les primes, transformons d'abord (2.22), en supposant que les primes futures seront égales à la dernière prime payée:

$$
\begin{aligned}
V_{p}(t)= & \left(\exp -\int_{t}^{N}\left(\delta(s)-\frac{1}{2} \sigma^{2}(s)\right) d s \cdot \exp -\int_{t}^{N} \sigma(s) d w(s)\right) \\
& -\bar{p}_{[t]} \cdot \sum_{j=[t]+1}^{N-1} \exp -\int_{t}^{j}\left(\delta(s)-\frac{\sigma^{2}(s)}{2}\right) d s \cdot \exp -\int_{t}^{j} \sigma(s) d w(s) .
\end{aligned}
$$

La tarification d'équilibre recherchée devra être telle que:

$$
V(j)=\mathbb{E}\left[V_{p}(j) \mid \mathscr{F}_{j}\right], \quad j=0,1, \ldots, N-1 .
$$


On a:

$$
\mathbb{E}\left(V_{p}(j) \mid \mathscr{F}_{j}\right)=\exp \int_{j}^{N}\left(\sigma^{2}(s)-\delta(s)\right) d s-\bar{p}_{j} \sum_{i=j+1}^{N-1} \exp \int_{j}^{i}\left(\sigma^{2}(s)-\delta(s)\right) d s .
$$

En exprimant l'égalité (2.23), il vient:

$$
\begin{aligned}
\bar{p}_{j}^{*}= & {\left[\exp \int_{j}^{N}\left(\sigma^{2}(s)-\delta(s)\right) d s-\sum_{i=0}^{j-1} \bar{p}_{i}^{*} \exp \int_{i}^{j}\left(\delta(s)-\frac{\sigma^{2}(s)}{2}\right) d s\right.} \\
& \left.\cdot \exp \int_{i}^{j} \sigma(s) d w(s)\right] /\left[\sum_{i=j}^{N-1} \exp \int_{j}^{i}\left(\sigma^{2}(s)-\delta(s)\right) d s\right]
\end{aligned}
$$

On remarque que ces primes sont bien mesurables par rapport à la filtration $\left\{\mathscr{F}_{t}\right\}$.

En particulier, pour $j=0$ :

$$
\bar{p}_{0}^{*}=\frac{\exp \int_{0}^{N}\left(\sigma^{2}(s)-\delta(s)\right) d s}{\sum_{j=0}^{N-1} \exp \int_{0}^{j}\left(\sigma^{2}(s)-\delta(s)\right) d s}
$$

En comparant à la prime classique (2.3), on remarque que $\bar{p}_{0}^{*}$ est calculée d'une manière déterministe aux tâux hors-risque $\delta-\sigma^{2}$.

Cette première prime est donc supérieure à la prime classique, puisqu'elle intègre le risque financier sur l'horizon $[0, N]$ :

$$
\bar{p}_{0}^{*} \geqslant p^{*} \text {. }
$$

Les primes ultérieures ne seront en général pas constantes, mais varieront en fonction des écarts stochastiques sur les taux d'intérêt, grâce à la formule de récurrence (2.24).

Dans cette formule, on remarque:

(a) pour les engagements futurs, on actualise grâce aux taux hors-risque $\delta-\sigma^{2}$;

(b) pour les réserves du passé, on capitalise grâce au facteur de capitalisation stochastique (1.7), i.e., en utilisant les taux réels.

Etudions à présent le comportement de la réserve au voisinage du terme du contrat.

Le dernier ajustement se fait en $t=N-1$; la dernière prime payée $\bar{p}_{N-1}^{*}$ est telle que:

$$
V(N-1)=\mathbb{E}\left(V_{p}(N-1) \mid \mathscr{F}_{N-1}\right)
$$

Or

$$
\begin{aligned}
\mathbb{E}\left(V_{p}(N-1) \mid \mathscr{F}_{N-1}\right) & =\mathbb{E}\left(\varphi_{N-1}(N) \mid \mathscr{F}_{N-1}\right) \\
& =\exp \int_{N-1}^{N}\left(\sigma^{2}(s)-\delta(s)\right) d s
\end{aligned}
$$


Donc

$$
V(N-1)=\exp \int_{N-1}^{N}\left(\sigma^{2}(s)-\delta(s)\right) d s .
$$

Cette réserve est déterministe et est égale à la réserve obtenue en cas de paiement par prime unique (cf. 2.17).

Etudions enfin l'évolution moyenne des primes.

De la formule (2.24), il vient:

$$
\mathbb{E} \bar{p}_{j}^{*}=\frac{\exp \int_{j}^{N}\left(\sigma^{2}(s)-\delta(s)\right) d s-\sum_{i=0}^{j-1} \mathbb{E} \bar{p}_{i}^{*} \cdot \exp \int_{i}^{j} \delta(s) d s}{\sum_{i=j}^{N-1} \exp \int_{j}^{i}\left(\sigma^{2}(s)-\delta(s)\right) d s}
$$

De même:

$$
\mathbb{E} \bar{p}_{j+1}^{*}=\frac{\exp \int_{j+1}^{N}\left(\sigma^{2}(s)-\delta(s)\right) d s-\sum_{i=0}^{j} \mathbb{E} \bar{p}_{i}^{*} \cdot \exp \int_{i}^{j+1} \delta(s) d s}{\sum_{i=j+1}^{N-1} \exp \int_{j+1}^{i}\left(\sigma^{2}(s)-\delta(s)\right) d s}
$$

En multipliant cette dernière expression haut et bas par exp $\int_{j}^{j+1}\left(\sigma^{2}(s)-\delta(s)\right)^{\dot{j}} d s$, il vient:

$$
\begin{aligned}
\mathbb{E} \bar{p}_{j+1}^{*}= & {\left[\mathbb{E} \bar{p}_{j}^{*} \sum_{i=j+1}^{N-1} \exp \int_{j}^{i}\left(\sigma^{2}(s)-\delta(s)\right) d s-\sum_{i=0}^{j} \mathbb{E} \bar{p}_{i}^{*} \exp \int_{i}^{j} \delta(s) d s\right.} \\
& \left.\cdot\left(\exp \int_{j}^{j+1} \sigma^{2}(s) d s-1\right)\right] /\left[\sum_{i=j+1}^{N-1} \exp \int_{j}^{i}\left(\sigma^{2}(s)-\delta(s)\right) d s\right]
\end{aligned}
$$

On a donc la formule générale de baisse moyenne des primes:

$$
\begin{aligned}
\mathbb{E} \bar{p}_{j+1}^{*} & =\mathbb{E} \bar{p}_{j}^{*}-\frac{\sum_{i=0}^{j} \mathbb{E} \bar{p}_{i}^{*} \cdot \exp \int_{i}^{j+1} \delta(s) d s\left(1-\exp \left(-\int_{j}^{j+1} \sigma^{2}(s) d s\right)\right)}{\sum_{i=j+1}^{N-1} \exp \int_{j+1}^{i}\left(\sigma^{2}(s)-\delta(s)\right) d s} \\
& =\mathbb{E} \bar{p}_{j}^{*}-\pi_{j+1} .
\end{aligned}
$$

La correction annuelle $\pi_{j+1}$ peut s'interpréter financièrement:

(a)

$$
\sum_{i=0}^{j} \mathbb{E} \bar{p}_{i}^{*} \exp \int_{i}^{j} \delta(s) d s
$$


représente la valeur capitalisée, de manière déterministe, en $t=j$, des primes moyennes passées.

(b)

$$
\begin{aligned}
\exp \int_{j}^{j+1} \delta(s) d s\left(1-\exp \left(-\int_{j}^{j+1} \sigma^{2}(s) d s\right)\right)= & \exp \int_{j}^{j+1} \delta(s) d s \\
& -\exp \int_{j}^{j+1}\left(\delta(s)-\sigma^{2}(s)\right) d s
\end{aligned}
$$

représente la différence entre les valeurs capitalisées entre $j$ et $j+1$, sous (c) les taux avec risque $\delta$, et les taux hors-risque $\delta-\sigma^{2}$.

$$
\sum_{i=0}^{j} \mathbb{E} \bar{p}_{i}^{*} \exp \int_{i}^{j+1} \delta(s) d s\left(1-\exp \left(-\int_{j}^{j+1} \sigma^{2}(s) d s\right)\right)
$$

peut donc s'interpréter comme le coût du risque financier entre $t=j$ et $t=j+1$ sur la réserve moyenne.

Ce coût ne doit donc plus être compris dans la prime payable en $t=j+1$, ainsi que dans les primes ultérieures.

(d) Cette économie est divisée par $\sum_{i=j+1}^{N-1} \exp \int_{j+1}^{i}\left(\sigma^{2}(s)-\delta(s)\right) d s$ i.e. elle est répartie de manière uniforme sur l'horizon $[j+1, N]$, sous les taux horsrisque $\delta-\sigma^{2}$.

La diminution graduelle des primes moyennes ne fait donc que refléter la réduction progressive de l'horizon d'incertitude financière.

\section{EQUATION DIFFERENTIELLE STOCHASTIQUE DE THIELE}

On généralise ici aux opérations viagères, les techniques stochastiques financières introduites au paragraphe 2 .

On se propose notamment d'obtenir une équation différentielle stochastique de la réserve mathématique des contrats d'assurance sur la vie.

\subsection{Théorie Classique}

On considère un contrat d'assurance sur la vie souscrit à l'âge $x$ pour une durée $N$, et prévoyant contre versement de primes, le paiement d'un capital $S_{x+\imath}$ en cas de décès à l'âge $x+t(t<N)$ et d'un capital vie $S$ en cas de vie à l'âge $x+N$.

Le taux d'intérêt $\delta$ est supposé constant.

La population de référence est modélisée par une loi de référence $\left\{l_{x}\right\}$, ( $l_{x}$ étant supposée continûment différentiable et monotone décroissante). Le taux 
instantané de mortalité est:

$$
\mu_{x}=-\frac{1}{l_{x}} \frac{d l_{x}}{d_{x}}
$$

En notant ${ }_{t} V_{x}$ la réserve mathématique en $t$ et $P_{x+t}$ la densité de prime, on montre (voir, DE VYLDER and JAUMAIN, 1976), que la réserve est solution d'une équation différentielle, appelée équation de Thiele:

$$
\frac{d_{t} V_{x}}{d t}={ }_{t} V_{x}\left[\mu_{x+t}+\delta\right]+P_{x+t}-S_{x+t} \mu_{x+t}
$$

\subsection{Théorie Stochastique}

Plutôt que d'être calculée au taux constant $\delta$, la réserve mathématique est capitalisée sous un flux wienérien:

$$
I(t)=\int_{0}^{t} \delta(s) d s+\int_{0}^{t} \sigma(s) d w(s) .
$$

De plus, la densité $P_{x+t}$ sera un processus stochastique, supposé adapté à la filtration $\left\{\mathscr{F}_{t}\right\}$.

De manière à obtenir dans ce cadre stochastique, l'équation de la réserve mathématique, considérons une réserve collective: $C(t)==l_{x+t} \cdot{ }_{t} V_{x}$

Cette réserve collective

(1) est capitalisée sous le flux wienérien $I$

(2) est alimentée par les primes: $l_{x+t} P_{x+t}$,

(3) est amputée des capitaux décès: $S_{x+t} d l_{x+t}$.

L'équation intégrale de cette réserve est donc:

$$
C(t)=C(0)+\int_{0}^{t} C(s) d I(s)+\int_{0}^{t} l_{x+s} P_{x+s} d s+\int_{0}^{t} S_{x+s} d l_{x+s}
$$

ou:

$$
d C(t)=C(t) \delta(t) d t+C(t) \sigma(t) d w(t)+l_{x+t} P_{x+t} d t+S_{x+t} d l_{x+t}
$$

En notant: $m(t)=l_{x+t} P_{x+t}+l_{x+t}^{\prime} S_{x+t}$, l'équation peut s'écrire:

$$
d C(t)=(C(t) \delta(t)+m(t)) d t+C(t) \sigma(t) d w(t)
$$

i.e. une équation différentielle stochastique linéaire, dont la solution peut se mettre sous la forme

$$
C(t)=\int_{0}^{t} C_{r}(t) m(r) d r
$$


où $C_{r}(t)$ est solution de l'équation homogène associée à (3.3):

$$
\begin{aligned}
d C_{r}(t) & =C_{r}(t) \delta(t) d t+C_{r}(t) \sigma(t) d w(t) \\
C_{r}(t) & =1 .
\end{aligned}
$$

$C_{r}(t)$ est donc le facteur de capitalisation obtenu au (1.7), et finalement:

$$
\begin{aligned}
C(t)= & \int_{0}^{t} \exp \int_{r}^{t}\left(\delta(s)-\frac{\sigma^{2}(s)}{2}\right) d s \\
& \cdot \exp \int_{r}^{t} \sigma(s) d w(s) \cdot\left(l_{x+r} P_{x+r}+l_{x+r}^{\prime} S_{x+r}\right) d r .
\end{aligned}
$$

La réserve mathématique individuelle est donnée par:

$$
{ }_{t} V_{x}=\frac{1}{l_{x+t}} C(t) .
$$

Puisque $l_{x+t}$ est une fonction déterministe, il vient en différentiant

$$
d_{t} V_{x}=\frac{1}{l_{x+t}} d C(t)+C(t) d\left(\frac{1}{l_{x+t}}\right)
$$

En utilisant (3.3), on a:

$$
\begin{aligned}
d_{t} V_{x}= & \frac{1}{l_{x+t}}\left[C(t) \delta(t) d t+C(t) \sigma(t) d w(t)+l_{x+t} P_{x+t} d t+S_{x+t} d l_{x+t}\right] \\
& -C(t) \frac{l_{x+t}^{\prime}}{l_{x+t}} d t
\end{aligned}
$$

En tenant compte de (3.1) et (3.5):

$$
d_{t} V_{x}=\left[{ }_{t} V_{x}\left(\delta(t)+\mu_{x+t}\right)+P_{x+t}-S_{x+t} \mu_{x+t}\right] d t+{ }_{t} V_{x} \sigma(t) d w(t)
$$

La réserve mathématique devient solution d'une équation différentielle stochastique, généralisant l'équation classique (3.2).

Dans ce modèle stochastique, le densité de prime peut être considérée comme un contrôle stochastique sur l'état du système (la réserve), solution d'une équation différentielle stochastique.

La solution de l'équation (3.6) s'écrit (cf. (3.5) et (3.4)):

$$
{ }_{i} V_{x}=\int_{0}^{t} \exp \int_{r}^{t}\left(\delta(s)-\frac{\sigma^{2}(s)}{2}\right) d s \exp \int_{r}^{t} \sigma(s) d w(s)\left(\frac{l_{x+r}}{l_{x+t}} P_{x+r}+\frac{l_{x+r}^{\prime}}{l_{x+t}} S_{x+r}\right) d r
$$




$$
\begin{aligned}
, V_{x}= & \int_{0}^{t} \exp \int_{r}^{t}\left(\delta(s)+\mu_{x+s}-\frac{\sigma^{2}(s)}{2}\right) d s \cdot \exp \int_{r}^{t} \sigma(s) d w(s) \\
& \cdot\left(P_{x+r}-\mu_{x+r} S_{x+r}\right) d r
\end{aligned}
$$

Cette réserve est une réserve rétrospective, égale à la différence entre la valeur capitalisée des primes payées et la valeur capitalisée des capitaux risque, la capitalisation se faisant sous le "flux viager":

$$
\bar{I}(t)=\int_{0}^{t}\left(\delta(s)+\mu_{x+s}\right) d s+\int_{0}^{t} \sigma(s) d w(s)
$$

Remarque. Comme pour les opérations de capitalisation, on peut introduire une réserve prospective égale à la différence entre la valeur actuelle des capitaux décès et vie futurs, et la valeur actuelle des primes à échoir. La densité de prime d'équilibre peut également être obtenue, en égalant à chaque instant réserve rétrospective et espérance conditionnelle de la réserve prospective.

Les relations obtenues au paragraphe 2 se généralisent naturellement.

\section{EXEMPLES NUMERIQUES}

Les notions de taux instantanés avec risque et hors risque permettent d'obtenir facilement une estimation des paramètres du modèle.

En particulier, si les fonctions $\delta$ et $\sigma$ sont constantes, on a:

taux instantané avec risque: $\quad \delta=\ln (1+i)$

taux instantané hors risque: $\quad \eta=\delta-\sigma^{2}=\ln (1+j)$.

Il suffit donc de se donner:

$$
\begin{aligned}
& i, \text { le taux annuel avec risque } \\
& j, \text { le taux annuel hors risque }
\end{aligned}
$$

On a alors:

$$
\begin{aligned}
\delta & =\ln (1+i) \\
\sigma^{2} & =\ln (1+i / 1+j) .
\end{aligned}
$$

Les exemples proposés portent sur la tarification et la technique de réservation stochastique des opérations de capitalisation. 
EXEMPLE 1. Taux avec risque: $5 \%$. Taux sans risque: $4 \%$. Durée du contrat: 10 ans.

\section{(a) Paiement par Prime Unique}

Prime unique déterministe: $P^{*}=0,61391$

Prime unique stochastique moyenne: $P=0,67556$

Evolution des réserves mathématiques:

\begin{tabular}{ccccc}
\hline \hline & $\begin{array}{c}\text { Réserve } \\
\text { Réserve } \\
\text { Déterministe } \\
t\end{array}$ & $\begin{array}{c}\text { Stochastique } \\
\text { Moyenne } \\
(1)\end{array}$ & $\begin{array}{c}\text { Réserve de } \\
\text { Risque } \\
(2)-(1)\end{array}$ & $\begin{array}{c}\% \\
(2)-(1) /(1)\end{array}$ \\
\hline 0 & 0,61391 & 0,67556 & 0,06165 & 10,04 \\
1 & 0,64461 & 0,70259 & 0,05798 & 8,99 \\
2 & 0,67684 & 0,73069 & 0,05385 & 7,96 \\
3 & 0,71068 & 0,75992 & 0,04924 & 6,93 \\
4 & 0,74622 & 0,79032 & 0,04410 & 5,91 \\
5 & 0,78353 & 0,82193 & 0,03840 & 4,90 \\
6 & 0,82270 & 0,85480 & 0,03210 & 3,90 \\
7 & 0,86384 & 0,88900 & 0,02516 & 2,91 \\
8 & 0,90703 & 0,92456 & 0,01753 & 1,93 \\
9 & 0,95238 & 0,96154 & 0,00916 & 0,96 \\
10 & 1 & 1 & 0 & 0 \\
\hline
\end{tabular}

(b) Paiement par Primes Annuelles:

Prime annuelle déterministe nivelée: $p^{*}=0,07572$

Evolution des primes annuelles stochastiques moyennes:

\begin{tabular}{lcc}
\hline \hline$t$ & $\begin{array}{c}\text { Prime } \\
\text { Moyenne }\end{array}$ & $\begin{array}{c}\text { Prime Moyenne/ } \\
\text { Prime Déterministe }\end{array}$ \\
\hline 0 & 0,08009 & 1,0577 \\
1 & 0,07998 & 1,0563 \\
2 & 0,07975 & 1,0532 \\
3 & 0,07935 & 1,0479 \\
4 & 0,07872 & 1,0396 \\
5 & 0,07777 & 1,0270 \\
6 & 0,07634 & 1,0081 \\
7 & 0,07411 & 0,9787 \\
8 & 0,07029 & 0,9283 \\
9 & 0,06172 & 0,8151 \\
\hline
\end{tabular}


Evolution des réserves mathématiques:

\begin{tabular}{|c|c|c|c|c|}
\hline$t$ & $\begin{array}{c}\text { Réserve } \\
\text { Déterministe }\end{array}$ & $\begin{array}{c}\text { Réserve } \\
\text { Stochastique } \\
\text { Moyenne }\end{array}$ & $\begin{array}{c}\text { Réserve de } \\
\text { Risque }\end{array}$ & $\%$ \\
\hline 0 & 0,07572 & 0,08009 & 0,00437 & 5,77 \\
\hline 1 & 0,15522 & 0,16408 & 0,00885 & 5,70 \\
\hline 2 & 0,23870 & 0,25203 & 0,01333 & 5,58 \\
\hline 3 & 0,32636 & 0,34398 & 0,01762 & 5,40 \\
\hline 4 & 0,41839 & 0,43989 & 0,02150 & 5,14 \\
\hline 5 & 0,51503 & 0,53965 & 0,02462 & 4,78 \\
\hline 6 & 0,61650 & 0,64297 & 0,02647 & 4,29 \\
\hline 7 & 0,72305 & 0,74922 & 0,02618 & 3,62 \\
\hline 8 & 0,83492 & 0,85697 & 0,02206 & 2,64 \\
\hline 9 & 0,95238 & 0,96154 & 0,00916 & 0,96 \\
\hline 10 & 1 & 1 & 0 & 0 \\
\hline
\end{tabular}

EXEMPLE 2. Taux avec risque: $5 \%$. Taux sans risque: $4 \%$. Durée du contrat: 20 ans.

\section{(a) Paiement par Prime Unique}

Prime unique déterministe: $P^{*}=0,37689$

Prime unique stochastique moyenne: $P=0,45639$

Evolution des réserves mathématiques:

\begin{tabular}{|c|c|c|c|c|}
\hline$t$ & $\begin{array}{c}\text { Réserve } \\
\text { Déterministe }\end{array}$ & $\begin{array}{c}\text { Réserve } \\
\text { Stochastique } \\
\text { Moyenne }\end{array}$ & $\begin{array}{c}\text { Réserve de } \\
\text { Risque }\end{array}$ & $\%$ \\
\hline 0 & 0,37689 & 0,45639 & 0,07950 & 21,09 \\
\hline 1 & 0,39573 & 0,47464 & 0,07891 & 19,94 \\
\hline 2 & 0,41552 & 0,49363 & 0,07811 & 18,80 \\
\hline 3 & 0,43630 & 0,51337 & 0,07708 & 17,67 \\
\hline 4 & 0,45811 & 0,53391 & 0,07580 & 16,55 \\
\hline 5 & 0,48102 & 0,55527 & 0,07425 & 15,44 \\
\hline 6 & 0,50507 & 0,57748 & 0,07241 & 14,34 \\
\hline 7 & 0,53032 & 0,60057 & 0,07025 & 13,25 \\
\hline 8 & 0,55684 & 0,62460 & 0,06776 & 12,17 \\
\hline 9 & 0,58468 & 0,64958 & 0,06490 & 11,10 \\
\hline 10 & 0,61391 & 0,67556 & 0,06165 & 10,04 \\
\hline 11 & 0,64461 & 0,70259 & 0,05798 & 8,99 \\
\hline 12 & 0,67684 & 0,73069 & 0,05385 & 7,96 \\
\hline 13 & 0,71068 & 0,75992 & 0,04924 & 6,93 \\
\hline 14 & 0,74622 & 0,79032 & 0,04410 & 5,91 \\
\hline 15 & 0,78353 & 0,82193 & 0,03840 & 4,90 \\
\hline 16 & 0,82270 & 0,85480 & 0,03210 & 3,90 \\
\hline 17 & 0,86384 & 0,88900 & 0,02516 & 2,91 \\
\hline 18 & 0,90703 & 0,92456 & 0,01753 & 1,93 \\
\hline 19 & 0,95238 & 0,96154 & 0,00916 & 0,96 \\
\hline 20 & 1 & 1 & 0 & 0 \\
\hline
\end{tabular}


(b) Paiement par Primes Annuelles

Prime annuelle déterministe nivelée: $p^{*}=0,02880$

Evolution des primes annuelles stochastiques moyennes:

\begin{tabular}{rcc}
\hline \hline \multicolumn{1}{c}{$t$} & $\begin{array}{c}\text { Prime } \\
\text { Moyenne }\end{array}$ & $\begin{array}{c}\text { Prime Moyenne/ } \\
\text { Prime Déterministe }\end{array}$ \\
\hline 0 & 0,03229 & 1,1211 \\
1 & 0,03227 & 1,1203 \\
2 & 0,03222 & 1,1185 \\
3 & 0,03214 & 1,1157 \\
4 & 0,03202 & 1,1118 \\
5 & 0,03187 & 1,1064 \\
6 & 0,03167 & 1,0995 \\
7 & 0,03142 & 1,0908 \\
8 & 0,03110 & 1,0799 \\
9 & 0,03072 & 1,0665 \\
10 & 0,03024 & 1,0500 \\
11 & 0,02966 & 1,0298 \\
12 & 0,02894 & 1,0048 \\
13 & 0,02805 & 0,9738 \\
14 & 0,02693 & 0,9348 \\
15 & 0,02548 & 0,8845 \\
16 & 0,02355 & 0,8174 \\
17 & 0,02081 & 0,7225 \\
18 & 0,01648 & 0,5721 \\
19 & 0,00739 & 0,2566 \\
\hline & &
\end{tabular}

Evolution des réserves mathématiques:

\begin{tabular}{rlccc}
\hline \hline \multicolumn{1}{c}{ Réserve } & $\begin{array}{c}\text { Réserve } \\
\text { Stochastique } \\
\text { Móterministe }\end{array}$ & $\begin{array}{c}\text { Réserve de } \\
\text { Risque }\end{array}$ & $\%$ \\
\hline 0 & 0,02880 & 0,03229 & 0,00349 & 12,11 \\
1 & 0,05905 & 0,06617 & 0,00713 & 12,07 \\
2 & 0,09080 & 0,10170 & 0,01090 & 12 \\
3 & 0,12414 & 0,13892 & 0,01477 & 11,90 \\
4 & 0,15915 & 0,17788 & 0,01873 & 11,77 \\
5 & 0,19591 & 0,21865 & 0,02273 & 11,60 \\
6 & 0,23451 & 0,26125 & 0,02674 & 11,40 \\
7 & 0,27504 & 0,30573 & 0,03069 & 11,15 \\
8 & 0,31759 & 0,35212 & 0,03452 & 10,87 \\
9 & 0,36227 & 0,40044 & 0,03816 & 10,53 \\
10 & 0,40919 & 0,45070 & 0,04151 & 10,14 \\
11 & 0,45845 & 0,50290 & 0,04444 & 9,69 \\
12 & 0,51018 & 0,55698 & 0,04681 & 9,17 \\
13 & 0,56449 & 0,61288 & 0,04839 & 8,57 \\
14 & 0,62152 & 0,67045 & 0,04893 & 7,87 \\
15 & 0,68139 & 0,72945 & 0,04806 & 7,05 \\
16 & 0,74427 & 0,78947 & 0,04520 & 6,07 \\
17 & 0,81028 & 0,84975 & 0,03947 & 4,87 \\
18 & 0,87960 & 0,90871 & 0,02911 & 3,31 \\
19 & 0,95238 & 0,96154 & 0,00916 & 0,96 \\
20 & 1 & 1 & 0 & 0 \\
\hline
\end{tabular}


EXEMPLE 3. L'exemple 2 montre que dans certains cas, on peut obtenir en cours de contrat des réductions de prime très importantes.

Ce phénomène peut même conduire, pour des taux importants, à la cessation prématurée du paiement des primes avant le terme du contrat, et à l'affectation d'un solde à une réserve de participation bénéficiaire, comme le montre l'exemple extrême suivant:

Taux avec risque: $10 \%$. Taux sans risque: $9 \%$. Durée du contrat: 20 ans.

Prime annuelle déterministe nivelée: 0,01587

Evolution des primes stochastiques moyennes:

\begin{tabular}{rrr}
\hline \multicolumn{1}{c}{ t } & $\begin{array}{c}\text { Prime } \\
\text { Moyenne }\end{array}$ & $\begin{array}{c}\text { Prime Moyenne/ } \\
\text { Prime Déterministe }\end{array}$ \\
\hline 0 & 0,01793 & 1,1298 \\
1 & 0,01791 & 1,1286 \\
2 & 0,01788 & 1,1262 \\
3 & 0,01781 & 1,1221 \\
4 & 0,01772 & 1,1164 \\
5 & 0,01760 & 1,1086 \\
6 & 0,01743 & 1,0983 \\
7 & 0,01723 & 1,0853 \\
8 & 0,01697 & 1,0689 \\
9 & 0,01664 & 1,0486 \\
10 & 0,01624 & 1,0233 \\
11 & 0,01574 & 0,9919 \\
12 & 0,01513 & 0,9529 \\
13 & 0,01435 & 0,9041 \\
14 & 0,01336 & 0,8419 \\
15 & 0,01208 & 0,7610 \\
16 & 0,01035 & 0,6520 \\
17 & 0,00788 & 0,4962 \\
18 & 0,00392 & 0,2471 \\
19 & $-0,00446$ & $-0,2810$ \\
\hline & & \\
& &
\end{tabular}

\section{CONCLUSION}

L'introduction d'une incertitude financière continue sur les taux d'intérêt, modélisée par une intégrale stochastique, a permis de mettre en évidence trois structures différentes de taux:

(5.1) Les taux réels stochastiques, agrégés dans un flux wienérien:

$$
I(t)=\int_{0}^{t} \delta(s) d s+\int_{0}^{t} \sigma(s) d w(s) .
$$

(5.2) Les taux instantanés moyens, déterministes, donnés par la fonction $\delta(t)$.

(5.3) les taux instantanés moyens hors risque, donnés par la fonction $\delta(t)-\sigma^{2}(t)$.

Les formules de tarification et de réservation obtenues dans ce travail, et basées sur la méthode des espérances conditionnelles, illustrent l'irréversibilité du phénomène de capitalisation: 
Les éléments financiers du passé sont capitalisés sous les taux réels stochastiques (5.1), ou, pour les formules moyennes, sous les taux moyens (5.2);

Les éléments financiers du futur sont actualisés sous les taux instantanés hors risque (5.3).

Cette actualisation effectuée à un taux inférieur au taux moyen, revient à pénaliser les produits financiers futurs, du fait de leur incertitude.

\section{BIBLIOGRAPHIE}

DE VyLDER, F. et JAUMAIN, C. (1976) Exposé moderne de la théorie mathématique des opérations viagères. Office des Assureurs de Belgique.

GinMAN, I. I. et SKOROHOD, A. V. (1972) Stochastic differential equations. Springer Verlag.

MAlliaris, A. G. et Brock, W. A. (1981) Stochastic methods in economics and Finance. NorthHolland.

POllard, J. H. (1971) On Fluctuating Interest Rates. Bulletin de l'Association Royale des Actuaires Belges, No. 66

SAADA, M. (1979) Traité pratique de mathématiques financières. Vuibert: Paris.

SChNIEPER, R. (1983) Risk Processes with Stochastic Discounting. Mitteilungen der Vereinigung schweizerischer Versicherungsmathematiker, 2.

Pierre Devolder

43, Boulevard Théo Lambert, B-1070 Bruxelles, Belgium 